\title{
Avaliação de competências relacionadas com a literacia de informação de estudantes do ensino superior
} Assessment of competences related with information literacy of students of the higher education

\author{
Evaluación de competencias relacionadas con la
} alfabetización informacional de los estudiantes de la educación superior

\author{
Fatima Passos Kanitar \\ Centro Federal de Educação Tecnológica Celso Suckow da Fonseca (Cefet/RJ), Rio de Janeiro/RJ - \\ Brasil \\ Maria João Loureiro \\ Universidade de Aveiro (UA), Aveiro - Portugal
}

\begin{abstract}
Resumo
A investigação que orienta este trabalho analisou competências de pesquisa, seleção e tratamento de informação científica, com recurso às TIC, entre estudantes da Universidade de Aveiro. Aplicou-se um questionário, no qual parte dos seus resultados é mostrada, em especial, os que dizem respeito ao uso das TIC no contexto de informação científica e às necessidades de formação desses estudantes nesse domínio. Pela técnica de estatística descritiva, ficou evidenciado não só que a maior parte dos estudantes indica saber utilizar ferramentas digitais, assim como têm a percepção de possuir essas competências. Contudo, há dificuldade perante atividades que demandam essa literacia. Dados deste e de outros estudos apontam a necessidade de inclusão dessa matéria no currículo.
\end{abstract}

Palavras-chave: Avaliação, Competências, Literacia de informação, Tecnologias da Informação e Comunicação, Pós-graduação

\begin{abstract}
The research that guides this work analysed skills of searching, selecting and treating scientific information, using ICT, among students of the Aveiro University. A questionnaire was applied and part of its results are shown, as an objective of this article, especially those related to the use of ICT in the context of scientific information, and to the needs of those students' training in this context. By descriptive statistics technique it was evident that most of the students indicates knowing how to use digital tools as they have the perception of possessing those skills; however, there is difficulty in activities that demand that literacy. Data of that and other studies indicate the need of inclusion of this matter in the curriculum.
\end{abstract}

Keywords: Assessment, Skills, Information literacy, Information and Communication Technologies, Post-graduation

Revista Educação Online, Rio de Janeiro, n. 29, set-dez 2018, p.61-85 


\section{Resumen}

La investigación que guía este trabajo analizó las competencias de investigación, selección y tratamiento de la información científica, utilizando las TIC, entre los estudiantes de la Universidad de Aveiro. Se aplicó un cuestionario y se muestra parte de sus resultados, como objetivo de este artículo, especialmente los relacionados con el uso de las TIC en el contexto de la información científica y las necesidades de la formación de esos estudiantes en ese dominio. Por la técnica de estadística descriptiva fue evidenciado que la mayor parte de los estudiantes no sólo indica saber usar las herramientas digitales sino que tienen la percepción de poseer esas competencias. Sin embargo, hay dificultad en actividades que necesitan esa alfabetización. Los datos de este y de otros estudios indican la necesidad de incluir ese tema en el plan de estudios.

Palabras clave: Evaluación, Competencias, Alfabetización informacional, Tecnologías de Información y Comunicación, Postgrado

\section{Introdução}

O presente artigo apresenta dados de uma tese de doutorado, que teve como objetivo a construção de estratégias de desenvolvimento de competências de pesquisa, seleção e tratamento de informação científica, com recurso às

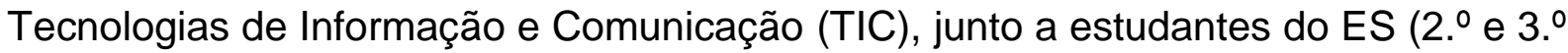
Ciclos de Bolonha ${ }^{1}$ ) de uma universidade portuguesa. Foi desenvolvido um referencial de avaliação, com a finalidade de obter os devidos critérios e indicadores (FIGARI, 1994; HADJI, 1994), tendo em vista a avaliação dessas competências.

Esse referencial é uma adaptação do trabalho de Timmers e Glass (2010) e Lopes e Pinto (2010), fundamentado na literatura consultada (HEAD, 2007; LAMPERT, 2005; LOPES; PINTO, 2010; REMPEL; DAVIDSON, 2008; VARGHESE, 2008; VIRKUS, 2009; WEILER, 2004) e contributos de bibliotecários da Universidade de Aveiro (UA). Sua confiabilidade é estabelecida pelos docentes/bibliotecários da área da educação e das TIC de Instituição de Ensino Superior (IES) de Portugal e do Brasil (AMADO, 2009). Essa credibilidade foi atingida pela caracterização dos profissionais que responderam ao questionário

\footnotetext{
${ }^{1}$ Os 2.. e 3. Ciclos de Bolonha correspondem, na mesma ordem, ao mestrado e doutorado no Brasil. O 1. Ciclo de Bolonha equivale ao curso de bacharelado.
}

Revista Educação Online, Rio de Janeiro, n. 29, set-dez 2018, p.61-85 
relacionado com a proposta inicial do referencial de avaliação. De igual modo, levouse em consideração não somente as suas indicações e comentários sobre o grau de relevância atribuído às competências relacionadas com a Literacia de Informação, mas também os contributos referentes à clarificação desse instrumento de coleta de dados e do seu formato eletrônico. Com base nesse instrumento usado com esses profissionais, foi desenvolvido um questionário, que foi aplicado aos 143 estudantes do 1. ano letivo do 2.. Ciclo e a 70 do 3. Ciclo do Departamento de Educação da UA.

Será apresentada a avaliação dos estudantes que participaram dessa pesquisa, de modo a caracterizar as competências que eles percepcionam possuir, e identificar suas necessidades de formação nesse domínio. Isso se justifica, por estudos semelhantes realizados nesse campo (HEAD, 2007; HEAD; MICHAEL B, 2009; KATZ, 2007; LAMPERT, 2005; REMPEL; DAVIDSON, 2008; SADLER; VARGHESE, 2008; WEILER, 2004), que indicam que a maior parte dos estudantes do ES não demonstra ter o conjunto de competências que integram o que se convencionou chamar de LI. Não se evidenciam as competências necessárias para dar o procedimento adequado à pesquisa de informação científica com uso da Internet, nomeadamente: seleção de diferentes fontes, avaliação da confiabilidade das informações obtidas, tratamento, organização, análise e "literacia de reprodução" (ESHET-ALKALAI, 2004). Desde a infância, os estudantes utilizam diferentes ambientes digitais, o que não assegura a aquisição das competências de LI (BOEKHORST, 2003).

No estudo aqui relatado, os estudantes portugueses do ES do Departamento de Educação da UA declaram que Ihes faltam determinadas competências relacionadas com a LI. Julgou-se ser de toda importância realizar o estudo com esses estudantes, devido ao fato de a maioria dos estudos encontrados sobre essa matéria se ocupar de estudantes do 1.․ Ciclo (CATTS; LAU, 2008; CONNER, 2012; ETS, 2006; HEAD, 2007; HEAD; MICHAEL B, 2009; HELMINEN; KATJIHINGUA, 2012; KATZ, 2007; LOPES; PINTO, 2010; PALNI, 2017; PEREIRA, 2011; PINTO, 2011; SALISBURY et al., 2012; SALISBURY; CORBIN; PESETA, 2013; ŠPIRANEC; KOS, 2013; TIMMERS; GLAS, 2010; TUAMSUK, 2013), ao invés dos 2.ํ e 3.․ Ciclos (CATTS; LAU, 2008; DUDZIAK, 2013; HELMINEN; KATJIHINGUA, 2012; 
MESQUITA, 2011; PINTO, 2011; REMPEL; DAVIDSON, 2008; SADLER; SILVA, 2013). Caracterizar as competências dos estudantes de ES para se ter 0 conhecimento das suas necessidades de formação vai ao encontro da cooperação da Unesco com o governo brasileiro, a qual visa ao aprimoramento do uso das TIC na educação do Brasil (UNESCO, 2017).

O uso e desenvolvimento das TIC favorecem uma mudança no modo de pesquisar, selecionar e tratar a informação científica (MACKEY; JACOBSON, 2011). A quantidade de páginas na Internet ultrapassa a ordem de bilhão, com um aumento na incerteza da sua qualidade, o que representa um grande desafio, pois não indica a criação de utilizadores mais competentes. A questão não é mais ter informação suficiente, é ter acesso a muita informação, com vários formatos e diversos níveis de qualidade (VIRKUS, 2014).

No contexto do ES, uma quantidade cada vez maior de usuários se beneficia da Internet para o ensino, aprendizagem e investigação (LAMPERT, 2005). Devido os recursos das TIC, é vital a valorização e aceitação da LI como uma necessidade verdadeira (MEULEMANS, 2002). Também se adicionam o questionamento, o pensamento crítico e a geração de conhecimento a ser aplicado em diversos contextos, que fazem parte do conjunto de competências indispensáveis do século XXI (AASL, 2007).

Diante do grande volume de informações científicas disponíveis na Internet e do seu alto potencial para a educação, faz-se necessário conhecer as necessidades de formação dos estudantes nessa área. Nesse sentido, questiona-se a ideia de que os mais jovens sabem tudo de que precisam saber para usufruir das oportunidades que a rede oferece ao estudo e à autoinstrução, visando ao desenvolvimento de atividades sistematizadas de Ensino e Aprendizagem (E/A) de literacia digital. Não existem competências sem saberes, as quais, na realidade, referem-se ao domínio prático de desenvolvimento de tarefas em diversas situações (PERRENOUD, 1998). Possuir um diploma não implica em ter competência nos diversos contextos em que são introduzidas inovações e saber gerir situações complexas e instáveis (LE BOTERF, 2005). Nesta investigação, a avaliação dessas competências foi adotada visando a oferecer subsídios para as ações nesse campo no meio acadêmico.

Revista Educação Online, Rio de Janeiro, n. 29, set-dez 2018, p.61-85 


\section{Objetivos e questões de investigação}

A investigação que dá sustenção aos argumentos apresentados neste artigo teve como objetivo formular hipóteses acerca das seguintes questões de investigação: Q1. Quais as percepções dos estudantes dos 2. e 3. Ciclos de Bolonha do Departamento de Educação da UA, em relação às suas competências de pesquisa, seleção e tratamento de informação científica, com recursos às TIC? Q2. Que necessidades de formação podem ser inferidas a partir dos resultados, considerando as percepções dos estudantes inquiridos a respeito das competências analisadas?

Levando em conta os objetivos e as questões de investigação, o estudo oferece dados que podem servir de referência para trabalhos futuros, visando à construção de estratégias para o desenvolvimento dessas competências entre estudantes, neste caso, estudantes de pós-graduação.

\section{Definição do instrumento de avaliação}

O instrumento de avaliação definido foi direcionado para o conhecimento das percepções dos estudantes com relação às suas competências associadas com as dimensões de pesquisa, seleção e tratamento de informação científica, assim como para a identificação de suas necessidades de formação.

Cabe explicitar o que é considerado para cada dimensão. As competências de pesquisa se relacionam com as ações adequadas para o acesso à informação científica, melhor dizendo, pela definição de um conjunto de fontes de informação, serviços e recursos on-line. As competências de seleção se prendem à avaliação de informação científica, ou seja, à aferição da qualidade e quantidade da informação recuperada na rede. Finalmente, o tratamento da informação científica recolhida pressupõe, ainda, sua indexação e organização, com uso das TIC, explorando, por exemplo, ferramentas da denominada cloud.

Para esta investigação, utiliza-se o termo competência, como capacidade de saber mobilizar; combinar/integrar e transpor recursos individuais incorporados (conhecimento, capacidade e atitudes), em quaisquer ambientes para a resolução de famílias de problemas (LE BOTERF, 2005). 


\subsection{Instrumento de avaliação}

Compreende-se que o instrumento de avaliação deve ser construído a partir da identificação, compreensão, sistematização e clarificação da informação encontrada (FERNANDES, 2008). Não se pode desatar qualquer tipo de avaliação de um sistema de referências, aqui representadas pelas dimensões estudadas, clarificadas pelos critérios (referente) e cada um deles exemplificados pelos indicadores (FIGARI, 1994; HADJI, 1994).

\subsubsection{Técnica de coleta de dados}

Fundamentado no exposto, foi desenvolvido um questionário, com base no referencial de avaliação, para a coleta de dados dos estudantes do $1 . .-$ ano letivo de pós-graduação do Departamento de Educação da UA (COHEN; MANION; MORRISON, 2005; QUIVY; CAMPENHOUDT, 2008). Foram contactados um total de 213 estudantes, isto é, $143(67,1 \%)$ do $2 .$. Ciclo e 70 (32,9\%) do 3.․ Ciclo. Houve um retorno de $35,2 \%$ de respostas, ou seja, $17,8 \%$ completas e $17,4 \%$ incompletas.

O Quadro 1 descreve a relação entre os objetivos e as questões utilizadas no questionário.

Quadro 1: Questões e objetivos do questionário

\begin{tabular}{|c|c|c|}
\hline $\begin{array}{l}\text { Questões de } \\
\text { investiqacão }\end{array}$ & $\begin{array}{l}\text { Perguntas do } \\
\text { questionário }\end{array}$ & Objetivos das perguntas \\
\hline \multirow{9}{*}{$\begin{array}{l}\text { Q1 - Quais as } \\
\text { percepções } \\
\text { dos } \\
\text { estudantes } \\
\text { dos } 2 . . \text { e e 3.․ } \\
\text { Ciclos de } \\
\text { Bolonha do } \\
\text { Departamento } \\
\text { de Educação } \\
\text { da UA em } \\
\text { relação às } \\
\text { suas } \\
\text { competências } \\
\text { de pesquisa, } \\
\text { seleção e } \\
\text { tratamento de } \\
\text { informação } \\
\text { científica com }\end{array}$} & $\begin{array}{l}\text { PRIMEIRA } \\
\text { PARTE }\end{array}$ & $\begin{array}{l}\text { 1. Conhecer o perfil dos estudantes de pós-graduação do } \\
\text { Departamento de Educação da UA. }\end{array}$ \\
\hline & $\begin{array}{l}\text { Questões 1, } 2 \text { e } \\
3\end{array}$ & $\begin{array}{l}\text { - Caracterizar os perfis pessoais quanto ao gênero, } \\
\text { nacionalidade e idade. }\end{array}$ \\
\hline & Questão 4 & $\begin{array}{l}\text { - Conhecer o programa do Departamento de } \\
\text { Educação da UA que os estudantes frequentam. }\end{array}$ \\
\hline & Questões 5 e 6 & $\begin{array}{l}\text { - Identificar a área de formação acadêmica inicial e } \\
\text { atividade profissional que exercem. }\end{array}$ \\
\hline & Questão 7 & $\begin{array}{l}\text { - Caracterizar o grau de familiaridade na utilização de } \\
\text { ferramentas das TIC. }\end{array}$ \\
\hline & Questão 8 & $\begin{array}{l}\text { - Identificar os dispositivos eletrônicos que costumam } \\
\text { usar. }\end{array}$ \\
\hline & $\begin{array}{l}\text { SEGUNDA } \\
\text { PARTE }\end{array}$ & $\begin{array}{l}\text { 2. Conhecer em que nível esses estudantes } \\
\text { percepcionam suas competências relacionadas com a LI } \\
\text { com recurso às TIC. }\end{array}$ \\
\hline & \multicolumn{2}{|c|}{ Dimensão Pesquisa de informação científica } \\
\hline & Questão C1 & $\begin{array}{l}\text { - Identificar uma necessidade de informação científica } \\
\text { na área da educação. }\end{array}$ \\
\hline
\end{tabular}

Revista Educação Online, Rio de Janeiro, n. 29, set-dez 2018, p.61-85 


\begin{tabular}{|c|c|c|}
\hline $\begin{array}{l}\text { Questões de } \\
\text { investigação }\end{array}$ & $\begin{array}{l}\text { Perguntas do } \\
\text { questionário }\end{array}$ & Objetivos das perguntas \\
\hline \multirow[t]{2}{*}{$\begin{array}{l}\text { recurso às } \\
\text { TIC? }\end{array}$} & Questão C2 & $\begin{array}{l}\text { - Construir uma estratégia de pesquisa de informação } \\
\text { científica. }\end{array}$ \\
\hline & Questão C3 & $\begin{array}{l}\text { - Utilizar técnicas/serviços de apoio à pesquisa de } \\
\text { informação científica na área de educação. }\end{array}$ \\
\hline \multirow{3}{*}{$\begin{array}{l}\text { Q2 - Que } \\
\text { necessidades } \\
\text { de formação } \\
\text { se podem }\end{array}$} & Questão C4 & - Pesquisar on-line literatura cinzenta. \\
\hline & Questão C5 & $\begin{array}{l}\text { - Utilizar fontes genéricas on-line de informação } \\
\text { científica. }\end{array}$ \\
\hline & \multicolumn{2}{|c|}{ Dimensão Seleção de informação científica } \\
\hline \multirow{8}{*}{$\begin{array}{l}\text { inferir } \\
\text { considerando } \\
\text { as } \\
\text { percepções } \\
\text { dos } \\
\text { estudantes } \\
\text { inquiridos a } \\
\text { respeito das } \\
\text { competências } \\
\text { analisadas? }\end{array}$} & Questão C6 & $\begin{array}{l}\text { - Avaliar a qualidade de fontes de informação } \\
\text { científica. }\end{array}$ \\
\hline & Questão C7 & $\begin{array}{l}\text { - Avaliar o grau de objetividade de informação } \\
\text { científica na área de educação. }\end{array}$ \\
\hline & \multicolumn{2}{|c|}{ Dimensão Tratamento de informação científica } \\
\hline & Questão C8 & - Organizar as informações científicas e suas fontes. \\
\hline & Questão C9 & - Caracterizar referências bibliográficas. \\
\hline & Questão C10 & - Resumir uma informação científica. \\
\hline & Questão C11 & $\begin{array}{l}\text { - Respeitar a propriedade intelectual de informação } \\
\text { científica consultada. }\end{array}$ \\
\hline & Questão C12 & $\begin{array}{l}\text { - Usar a informação científica sem a influência de } \\
\text { valores ou crenças. }\end{array}$ \\
\hline
\end{tabular}

Fonte: Autora

O Quadro 1 evidencia os objetivos de cada pergunta apresentada aos estudantes. Na primeira parte, quanto ao perfil dos estudantes, solicitou-se que informassem o gênero, a nacionalidade e a idade (questões 1, 2 e 3). Obteve-se o conhecimento do ciclo de estudos e curso que eles frenquentam no Departamento de Educação da UA (Questão 4), pois se tratando do 2. Ciclo têm-se: i) Ciências da Educação, ii) Didática, iii) Supervisão e iv) Promoção da leitura e bibliotecas escolares. No 3.ำ Ciclo, são assegurados os seguintes cursos: i) Psicologia, ii) Didática e Formação, iii) Multimédia em Educação e iv) Educação. Indicaram a área de formação acadêmica inicial (Questão 5) e a atividade profissional que desempenham (Questão 6).

Solicitou-se que apontassem o grau de familiaridade/experiência (Questão 7), numa escala de 1 a 5 (em que 1 corresponde a Não conheço, 2 a Conheço, mas não utilizo, 3 a utilizo de forma básica, 4 a Utilizo bem e 5 a Utilizo muito bem), relativamente ao uso das seguintes ferramentas das TIC: i) Agregadores de feeds (p. ex. Netvibes ou iGoogle), ii) Blogues, iii) Escrita colaborativa (p. ex. Wikis), iv) Office online (p. ex. Google Docs), v) Partilha de apresentações de vídeo (p. ex. Revista Educação Online, Rio de Janeiro, n. 29, set-dez 2018, p.61-85 
knovio), vi) Partilha de ficheiros (p. ex. Dropbox), vii) Partilha de fotos (p. ex. Flickr), viii) Partilha de slides (p. ex. Slideshare), ix) Partilha de vídeo ou áudio (p. ex. Youtube), x) Redes sociais (p. ex. Facebook, Twitter, Linkedin...) e xi) Social Bookmarking (p. ex. Delicious, Diigo).

Também foi pedido (Questão 8) que indicassem o(s) dispositivo(s) que mais utilizam da seguinte listagem: i) Desktop computer; ii) Laptop, iii) Tablet e iv) Smartphone. No caso de utilizarem outro tipo de dispositivo, deveriam informar qual. Desse modo, identificaram-se os estudantes, caracterizando-os, através dos dados recolhidos da primeira parte do questionário (COHEN; MANION; MORRISON, 2005).

$\mathrm{Na}$ segunda parte do questionário (Quadro 1), foi pedido que os estudantes indicassem o seu nível de competência, relativamente a todos os indicadores que integram os critérios relacionados com as dimensões pesquisa, seleção e tratamento de informação científica com recurso às TIC (Figura 1). Foi solicitado que indicassem, numa escala de 1 a 4 , o que melhor retratava sua competência relativa às atividades (1. Muito baixo, 2. Baixo, 3. Elevado, 4. Muito elevado). Caso não se sentissem seguros para responder, assinalassem a opção NS (Não Sei). Deve-se levar em conta que, quando se questionam atitudes, opiniões, gostos ou grau de satisfação, segundo Hill e Hill (2009), cinco respostas alternativas são suficientes para uma dada pergunta. Entretanto, é preciso ter cautela quanto à obtenção de resultados que não mostram a realidade, pois, quando há uma opção que seja neutra no meio da escala, os inquiridos tendem a indicá-la, supondo que ela é a mais segura, por não terem que mostrar uma opinião positiva ou negativa acerca do que é questionado. Considerando-se o exposto e procurando evitar que os estudantes deixassem de responder algumas questões na segunda parte do inquérito, foi apresentada a opção NS (Não Sei). A fim de evitar fraudes das respostas, elas não foram consideradas obrigatórias.

Desse modo, de forma resumida, na primeira parte, foram apresentadas oito questões para se conhecer o perfil dos estudantes inquiridos. Na segunda parte, visando a responder as questões de investigação, foram apresentadas 12 questões referentes às dimensões de pesquisa (5), seleção (2) e tratamento de informação científica (5). Em outras palavras, cada pergunta se relaciona com um dos seus 12 critérios, possuindo, na sua totalidade, 47 indicadores. Dessa maneira, obteve-se 
fundamento para análise das primeira e segunda questões de investigação (BODGAN; BIKLEN, 2013).

\section{Análise e discussão dos resultados}

Nesta seção serão apresentadas a análise e discussão dos resultados, em interlocução com a literatura consultada. Será apresentada, em primeiro lugar, a análise dos dados feita através da técnica de estatística descritiva (REIS, 2008). A coleta, administração e a interpretação dos dados recolhidos teve como base os critérios e indicadores, definidos no referencial de avaliação, mostrados na Figura 1 (QUIVY; CAMPENHOUDT, 2008; REIS, 2008). Foram feitas referências aos percentuais das frequências relativas dos níveis de competência que os estudantes consideram possuir e dos que indicam que não se sentem seguros para responder a respeito de suas competências.

Houve uma reflexão teórica a partir desses dados, apresentados em gráficos, assegurando a coerência e sentido do estudo (QUIVY; CAMPENHOUDT, 2008). Obtiveram-se, desse modo, subsídios para responder as duas questões de investigação, referidas à percepção que os estudantes do ES possuem a respeito de suas competências relacionadas com a LI, com recurso às TIC e das suas necessidades de formação nesse domínio.

Serão apresentadas a descrição e a caracterização das competências dos estudantes que participaram da pesquisa, bem como formuladas hipóteses e inferências analítico-reflexivas emitidas da percepção desses estudantes em relação as suas competências. Para tal, foram considerados os dados dos 47 indicadores (I1 ao 147), isto é, Pesquisa: I1 ao I3 para o critério C1, I4 ao I10 para C2, I11 ao I18 para C3, I19 ao I21 para C4 e I22 ao I23 para C5; Seleção: I24 ao I29 para C6 e I30 ao I31 para C7; Tratamento: I32 ao I35 para C8, I36 ao I40 para C9, I41 ao I43 para C10, I44 ao 145 para C11e 146 ao 147 para C12. Observe-se a Figura 1, que mostra trecho do questionário.

Revista Educação Online, Rio de Janeiro, n. 29, set-dez 2018, p.61-85 
Conhecer em que nivel os estudantes do ES da UA percepcionam suas competências relacionadas com a $L / \mathrm{com}$ recurso às $T I C$

\begin{tabular}{|c|c|c|c|c|c|}
\hline \multirow{2}{*}{\multicolumn{6}{|c|}{ 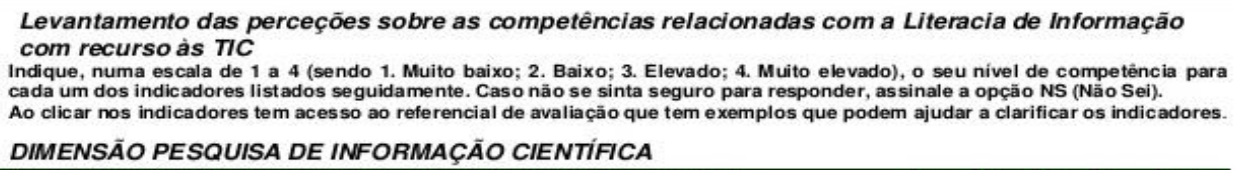 }} \\
\hline & & & & & \\
\hline C1. Definir com proficiência uma necessidade de informação cientifica: & 1 & 2 & 3 & 4 & NS \\
\hline \multicolumn{6}{|l|}{ 11. Identificar uma necessidade de informação científica. } \\
\hline \multicolumn{6}{|l|}{$\begin{array}{l}\text { 12. Definir os tópicos que se articulam com a necessidade de informação científica } \\
\text { identificada. }\end{array}$} \\
\hline \multicolumn{6}{|l|}{ DIMENSÃO SELEÇÃO DE INFORMAÇÃO CIENTIFICA } \\
\hline $\begin{array}{l}\text { C7. Examinar com proficiência o grau de objetividade da informação clentífica } \\
\text { disponibilizada. }\end{array}$ & 1 & 2 & 3 & 4 & NS \\
\hline \\
\hline $\begin{array}{l}\text { 131. Identificar se a informação científica é de pesquisa patrocinada por alguma } \\
\text { associação e, neste caso, se é permitida a divulgação dos dados obtidos. }\end{array}$ & & & & & \\
\hline \multicolumn{6}{|l|}{ DIMENSÃO TRATAMENTO DE INFORMAÇÃO CIENTIFICA } \\
\hline C12. Usar a infor mação cientifica sem a influência de valores ou crenças. & 1 & 2 & 3 & 4 & NS \\
\hline \multicolumn{6}{|l|}{ 146. Evitar referências influenciadas por valores pessoais ou crenças. } \\
\hline $\begin{array}{l}\text { 47. Ter disposição para alterar um conjunto interno de informaçōes/experiências, nāo } \\
\text { sendo inflexivel perante novas propostas. }\end{array}$ & & & & & \\
\hline
\end{tabular}

Figura 1 - Trecho do questionário aplicado aos ES do Departamento de Educação da UA

Com vista nos objetivos de cada questão, como mostrados no Quadro 1, os Quadros 2, 3, 4 e 5 apresentam a análise das respostas dos estudantes que contribuíram para esta investigação.

Quadro 2 - Perfil dos estudantes respondentes dos 2.. e 3. Ciclos de Bolonha do Departamento de Educação da UA

\begin{tabular}{|c|c|c|}
\hline $\begin{array}{l}\text { Questões de } \\
\text { investigação }\end{array}$ & $\begin{array}{l}\text { Perguntas } \\
\text { do } \\
\text { questionário }\end{array}$ & Análise das respostas \\
\hline \multirow{4}{*}{$\begin{array}{l}\text { Q1 - Quais as } \\
\text { percepções dos } \\
\text { estudantes dos } \\
2 .^{\circ} \text { e 3.․ Ciclos } \\
\text { de Bolonha do } \\
\text { Departamento } \\
\text { de Educação } \\
\text { da UA em } \\
\text { relação às suas } \\
\text { competências } \\
\text { de pesquisa, } \\
\text { seleção e } \\
\text { tratamento de } \\
\text { informação } \\
\text { científica com } \\
\text { recurso às TIC? }\end{array}$} & $\begin{array}{l}\text { PRIMEIRA } \\
\text { PARTE }\end{array}$ & $\begin{array}{l}\text { 1. Conhecer o perfil dos estudantes de pós-graduação do } \\
\text { Departamento de Educação da UA. }\end{array}$ \\
\hline & $\begin{array}{l}\text { Questões 1, } \\
2 \text { e } 3\end{array}$ & $\begin{array}{l}\text { - A maior parte dos estudantes é do gênero feminino } \\
(81,6 \%) \text { e de nacionalidade portuguesa }(94,7 \%) \text {. } \\
\text { Quase metade tem menos de } 30 \text { anos }(44,7 \%) \text { e os } \\
\text { demais se encontram entre } 30 \text { e } 49 \text { anos }(55,3 \%) \text {. }\end{array}$ \\
\hline & Questão 4 & $\begin{array}{l}\text { - A metade dos estudantes se acha matriculada no 2.0 } \\
\text { Ciclo (50\%) e, do mesmo modo, no } 3.0 \text { Ciclo (50\%). No } \\
2 . . \text { Ciclo, } 31,6 \% \text { estão no curso de Ciências da } \\
\text { Educação, } 15,8 \% \text { na Didática e } 2,6 \% \text { dos estudantes } \\
\text { não informaram o curso que se encontram } \\
\text { matriculados. No } 3 .- \text { Ciclo, } 21,1 \% \text { dos estudantes } \\
\text { estão no curso de Didática e Formação, } 7,8 \% \text { em } \\
\text { Multimídia em Educação e } 21,1 \% \text { em Educação. }\end{array}$ \\
\hline & $\begin{array}{l}\text { Questões } 5 \text { e } \\
6\end{array}$ & $\begin{array}{l}\text { - } 84,2 \% \text { possuem formação acadêmica inicial em } \\
\text { licenciatura na área de educação, os demais apontam } \\
\text { ter formação em arquitetura, psicologia e enfermagem. } \\
92,1 \% \text { indicam trabalhar na área da educação, como } \\
\text { docentes do ensino superior, secundário, pré-escolar, }\end{array}$ \\
\hline
\end{tabular}

Revista Educação Online, Rio de Janeiro, n. 29, set-dez 2018, p.61-85 


\begin{tabular}{|c|c|c|}
\hline $\begin{array}{l}\text { Questões de } \\
\text { investigação }\end{array}$ & $\begin{array}{l}\text { Perguntas } \\
\text { do } \\
\text { questionário } \\
\end{array}$ & Análise das respostas \\
\hline \multirow{3}{*}{$\begin{array}{l}\text { Q2 - Que } \\
\text { necessidades } \\
\text { de formação se } \\
\text { podem inferir, } \\
\text { considerando } \\
\text { as percepções } \\
\text { dos estudantes } \\
\text { inquiridos a } \\
\text { respeito das } \\
\text { competências } \\
\text { analisadas? }\end{array}$} & & $\begin{array}{l}\text { educação especial e 1. Ciclo do ensino básico. Os } \\
\text { que não ocupam a função de docente e trabalham } \\
\text { como enfermeiro, técnico de investigação e bolsista de } \\
\text { investigação. }\end{array}$ \\
\hline & Questão 7 & 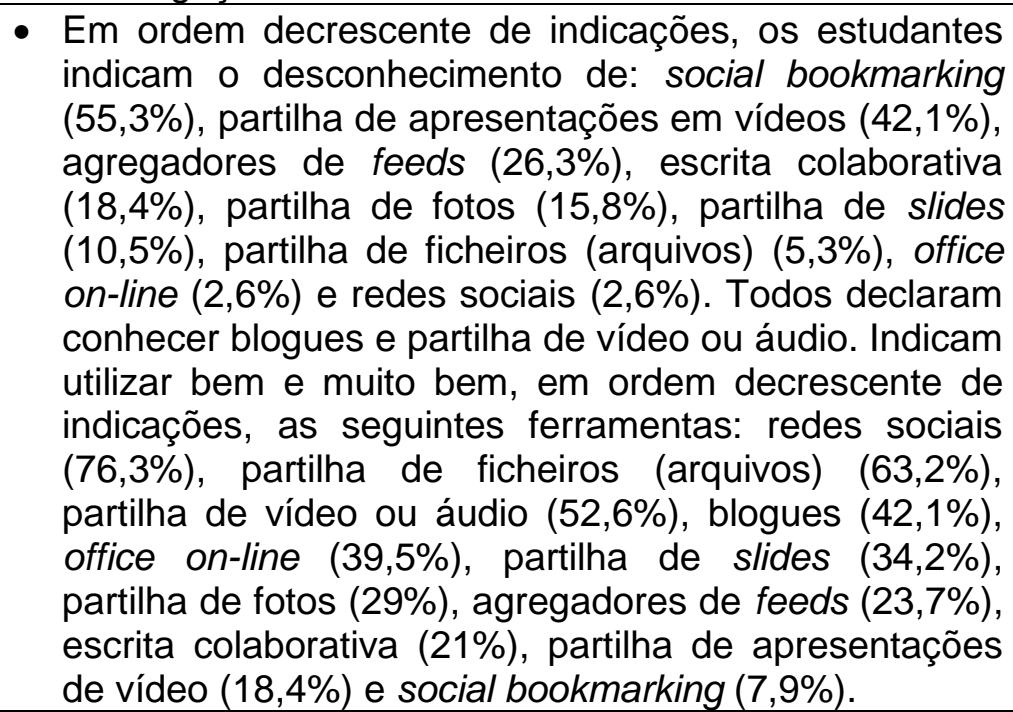 \\
\hline & Questão 8 & $\begin{array}{l}\text { - Em relação aos dispositivos eletrônicos, a maior parte } \\
\text { dos estudantes (60,5\%) utiliza desktop computer e } \\
\text { também laptop. Em menor escala, usam smartphones } \\
(31,6 \%) \text { e tablets }(18,4 \%) \text {. }\end{array}$ \\
\hline
\end{tabular}

A caracterização dos estudantes que responderam ao questionário é apresentada no Quadro 2. Deve-se ter em conta, de acordo com a resposta da Questão 7, apesar de os estudantes apontarem saber utilizar bem e muito bem determinadas ferramentas das TIC, não significa que tenham competências aqui estudadas (KATZ, 2007).

Quadro 3 - Percepção das competências de Pesquisa de informação científica dos estudantes respondentes dos $2 . .^{\circ}$ e $3 .$. Ciclos de Bolonha do Departamento de Educação da UA

\begin{tabular}{|l|l|l|}
\hline \multicolumn{1}{|c|}{$\begin{array}{c}\text { Questões de } \\
\text { investigação }\end{array}$} & $\begin{array}{c}\text { Perguntas } \\
\text { do } \\
\text { questionário }\end{array}$ & \multicolumn{1}{c|}{ Análise das respostas } \\
\hline $\begin{array}{l}\text { Q1 - Quais as } \\
\text { percepções dos } \\
\text { estudantes dos }\end{array}$ & $\begin{array}{l}\text { SEGUNDA } \\
\text { PARTE }\end{array}$ & $\begin{array}{l}\text { 2. Conhecer em que nível esses estudantes percebem } \\
\text { suas competências relacionadas com a LI com recurso } \\
\text { às TIC. }\end{array}$ \\
\cline { 2 - 3 } $\begin{array}{l}\text { Bolonha do } \\
\text { Departamento de }\end{array}$ & Dimensão Pesquisa de informação científica \\
\cline { 2 - 3 } $\begin{array}{l}\text { Educação da UA } \\
\text { em relação às }\end{array}$ & Questão C1 & $\begin{array}{l}\text { A maior parte dos estudantes tem percepção de suas } \\
\text { competências, relativamente a todos os indicadores } \\
\text { (I1 ao I3), a nível elevado, seguindo-se por muito } \\
\text { elevado. }\end{array}$ \\
\hline \hline
\end{tabular}

Revista Educação Online, Rio de Janeiro, n. 29, set-dez 2018, p.61-85 


\begin{tabular}{|c|c|c|}
\hline $\begin{array}{l}\text { Questões de } \\
\text { investigação }\end{array}$ & $\begin{array}{l}\text { Perguntas } \\
\text { do } \\
\text { questionário }\end{array}$ & Análise das respostas \\
\hline \multirow{4}{*}{$\begin{array}{l}\text { suas } \\
\text { competências de } \\
\text { pesquisa, seleção } \\
\text { e tratamento de } \\
\text { informação } \\
\text { científica com } \\
\text { recurso às TIC? } \\
\text { Q2 - Que } \\
\text { necessidades de } \\
\text { formação se } \\
\text { podem inferir } \\
\text { considerando as } \\
\text { percepções dos } \\
\text { estudantes } \\
\text { inquiridos a } \\
\text { respeito das } \\
\text { competências } \\
\text { analisadas? }\end{array}$} & Questão C2 & $\begin{array}{l}\text { - A maioria dos estudantes tem a percepção de suas } \\
\text { competências, relativamente a todos os indicadores } \\
\text { (14 ao I10), a nível elevado, seguindo-se por muito } \\
\text { elevado. }\end{array}$ \\
\hline & Questão C3 & $\begin{array}{l}\text { - O maior número dos estudantes indicam suas } \\
\text { competências em nível elevado, relativamente a } 5 \\
\text { indicadores (I14, I15, I16, I17 e I18), seguindo por } \\
\text { indicar nível muito elevado. De forma inversa, dois } \\
\text { indicadores (I11 e I12) são mais sinalizados para nível } \\
\text { muito elevado, seguindo-se ao elevado. A mesma } \\
\text { quantidade de estudantes aponta suas competências } \\
\text { em nível elevado e muito elevado em referência a um } \\
\text { indicador (I13). }\end{array}$ \\
\hline & Questão C4 & $\begin{array}{l}\text { - Dos três indicadores, a maior parte de sinalizações é } \\
\text { para nível elevado, seguindo-se muito elevado (I20 e } \\
\text { I21). Somente um indicador apresenta mais } \\
\text { indicações para nível muito elevado de competência } \\
\text { (I19). }\end{array}$ \\
\hline & Questão C5 & $\begin{array}{l}\text { - A maior parte dos estudantes tem a percepção de } \\
\text { suas competências, relativamente a todos os } \\
\text { indicadores, em nível elevado, seguindo-se por muito } \\
\text { elevado (I22 e I23). }\end{array}$ \\
\hline
\end{tabular}

De acordo com o Quadro 3, mais de 50\% dos estudantes do ES afirmam possuir nível elevado e muito elevado de competências. Não houve indicação de nível muito baixo para os indicadores 15 e 17 de C2, I12 ao 116 de C3, todos os indicadores de C4 e 122 de C5. Para todos os critérios (C1 ao C5) e seus indicadores (I1 ao I23), poucos estudantes admitiram possuir nível muito baixo, baixo ou não se sentem seguros para responder.

Quadro 4 - Percepção das competências de Seleção de informação científica dos estudantes respondentes dos $2 .^{\circ}$ e $3 .^{\circ}$ Ciclos de Bolonha do Departamento de Educação da UA

\begin{tabular}{|c|c|c|}
\hline Questões de investigação & $\begin{array}{l}\text { Perguntas } \\
\text { do } \\
\text { questionário }\end{array}$ & Análise das respostas \\
\hline \multirow{3}{*}{$\begin{array}{l}\text { Q1 - Quais as percepções } \\
\text { dos estudantes dos } 2 .^{\circ} \mathrm{e} \\
\text { 3.․ Ciclos de Bolonha do } \\
\text { Departamento de } \\
\text { Educação da UA, em } \\
\text { relação às suas } \\
\text { competências de pesquisa, } \\
\text { seleção e tratamento de }\end{array}$} & $\begin{array}{l}\text { SEGUNDA } \\
\text { PARTE }\end{array}$ & $\begin{array}{l}\text { 2. Conhecer em que nível esses estudantes } \\
\text { percebem suas competências relacionadas } \\
\text { com a LI com recurso às TIC. }\end{array}$ \\
\hline & \multicolumn{2}{|c|}{ Dimensão Seleção de informação científica } \\
\hline & Questão C6 & $\begin{array}{l}\text { - A maioria dos estudantes assinala possuir } \\
\text { nível de competências muito elevado e em } \\
\text { seguida elevado (I24, I27 e I29). Em } \\
\text { relação aos outros indicadores (I25, I26 e }\end{array}$ \\
\hline
\end{tabular}

Revista Educação Online, Rio de Janeiro, n. 29, set-dez 2018, p.61-85 


\begin{tabular}{|c|c|c|}
\hline Questões de investigação & $\begin{array}{c}\text { Perguntas } \\
\text { do } \\
\text { questionário }\end{array}$ & Análise das respostas \\
\hline \multirow{2}{*}{$\begin{array}{l}\text { informação científica com } \\
\text { recurso às TIC? } \\
\text { Q2 - Que necessidades de } \\
\text { formação se podem inferir, } \\
\text { considerando as } \\
\text { percepções dos estudantes } \\
\text { inquiridos a respeito das } \\
\text { competências analisadas? }\end{array}$} & & $\begin{array}{l}\text { I28), maior foi a quantidade que indicou } \\
\text { possuir grau elevado e logo a seguir muito } \\
\text { elevado. Diversos estudantes indicam grau } \\
\text { baixo de competência para I } 24 \text {. }\end{array}$ \\
\hline & Questão C7 & $\begin{array}{l}\text { - Para todos os indicadores } 130 \text { e I31, na } \\
\text { mesma ordem, um número aproximado e } \\
\text { igual de estudantes percebem possuir nível } \\
\text { muito elevado e baixo de competências. }\end{array}$ \\
\hline
\end{tabular}

Conforme o Quadro 4, considerando todos os indicadores (C6 e C7), percebem-se mais sinalizações para nível elevado (de 26,3\% a 50\%), muito elevado (de $23,7 \%$ a $47,4 \%$ ) e baixo (de $7,9 \%$ a $23,7 \%$ ). Em seguida, são as sinalizações dos estudantes que não se sentem seguros (de 5,3\% a 18,4\%) para responder e, em menor escala, são as indicações para nível muito baixo (de 0\% a 7,9\%); 23,4\% dos estudantes indicam ter nível baixo para 124 do critério C6. Para I31 do C7, é igual à quantidade de estudantes (26,3\%) que considera ter nível baixo e muito elevado de competências. E ainda com vista ao C7, para o 130, é próxima a quantidade de estudantes que indicam ter nível baixo $(26,3 \%)$ e muito elevado $(23,7 \%)$ de competências. Não houve sinalizações para muito baixo em relação aos indicadores I27, 128 e 129 de C6 e para 130 e I31 de C7.

Quadro 5 - Percepção das competências de Tratamento de informação científicados estudantes respondentes dos 2.ํ e 3. Ciclos de Bolonha do Departamento de Educação da UA

\begin{tabular}{|c|c|c|}
\hline $\begin{array}{l}\text { Questões de } \\
\text { investigação }\end{array}$ & $\begin{array}{l}\text { Perguntas } \\
\text { do } \\
\text { questionário }\end{array}$ & Análise das respostas \\
\hline \multirow{5}{*}{$\begin{array}{l}\text { Q1 - Quais as } \\
\text { percepções dos } \\
\text { estudantes dos 2.. e 3.. } \\
\text { Ciclos de Bolonha do } \\
\text { Departamento de } \\
\text { Educação da UA, em } \\
\text { relação às suas } \\
\text { competências de } \\
\text { pesquisa, seleção e } \\
\text { tratamento de } \\
\text { informação científica } \\
\text { com recurso às TIC? } \\
\text { Q2 - Que necessidades }\end{array}$} & $\begin{array}{l}\text { SEGUNDA } \\
\text { PARTE }\end{array}$ & $\begin{array}{l}\text { 2. Conhecer em que nível esses estudantes } \\
\text { percebem suas competências relacionadas com } \\
\text { a LI com recurso às } T I C \text {. }\end{array}$ \\
\hline & \multicolumn{2}{|c|}{ Dimensão Tratamento de informação científica } \\
\hline & Questão C8 & $\begin{array}{l}\text { - Mais da metade dos estudantes percebe } \\
\text { possuir nível elevado e muito elevado de } \\
\text { competências para I32 e I35. De forma } \\
\text { próxima e igual, indicam ter nível de } \\
\text { competências muito elevado e baixo, } \\
\text { respectivamente, para I33 e I34. }\end{array}$ \\
\hline & Questão C9 & $\begin{array}{l}\text { - Maior é a quantidade de estudantes que } \\
\text { aponta suas competências em nível elevado } \\
\text { e muito elevado. }\end{array}$ \\
\hline & Questão C10 & - A maioria dos estudantes percebe suas \\
\hline
\end{tabular}

Revista Educação Online, Rio de Janeiro, n. 29, set-dez 2018, p.61-85 


\begin{tabular}{|c|c|c|}
\hline $\begin{array}{l}\text { Questões de } \\
\text { investigação }\end{array}$ & $\begin{array}{l}\text { Perguntas } \\
\text { do } \\
\text { questionário }\end{array}$ & Análise das respostas \\
\hline \multirow{3}{*}{$\begin{array}{l}\text { de formação podem-se } \\
\text { inferir, considerando as } \\
\text { percepções dos } \\
\text { estudantes inquiridos a } \\
\text { respeito das } \\
\text { competências } \\
\text { analisadas? }\end{array}$} & & $\begin{array}{l}\text { competências em nível elevado e muito } \\
\text { elevado. }\end{array}$ \\
\hline & Questão C11 & $\begin{array}{l}\text { - Mais da metade dos estudantes indica ter } \\
\text { competências em nível elevado e muito } \\
\text { elevado. }\end{array}$ \\
\hline & Questão C12 & $\begin{array}{l}\text { - A maior parte dos estudantes assinala suas } \\
\text { competências em nível elevado e muito } \\
\text { elevado. }\end{array}$ \\
\hline
\end{tabular}

Conforme o Quadro 5, a maior parte dos estudantes do ES (> 50\%) percebe suas competências de tratamento de informação científica, com recurso às TIC, em nível elevado e muito elevado, para 132 e 135 do C8 e, do mesmo modo, para todos os indicadores do C9 ao C12. Entretanto, para C8, é idêntica a quantidade de estudantes $(28,9 \%)$ que aponta ter nível baixo e muito elevado de competência para o indicador 134 e, de forma igual, é muita próxima a quantidade de estudantes que aponta o mesmo nível de competências para baixo $(23,7 \%)$ e muito elevado $(21,1 \%)$ em relação à 133 . Para todos os indicadores do $\mathrm{C} 9$ ao $\mathrm{C} 12$, e ainda para $\mathrm{C} 8$ considerando 132 e I35, poucos estudantes afirmam possuir nível baixo, muito baixo ou não se sentem seguros para responder. Não houve indicações de nível muito baixo para os indicadores I36, I37, I38 e 140 de C9, e menos de 3\% apontam não se sentirem seguros em relação a todos os seus indicadores (I36 ao 140).

\subsection{Necessidades de formação dos estudantes do ES}

Com a finalidade de obter fundamento para a necessidade de formação dos estudantes do ES, pela leitura cuidadosa da análise dos resultados, foi considerada a quantidade de estudantes que percebe suas competências em nível baixo, superior a muito elevado, com a menor quantidade de sinalizações para muito elevado: o que aconteceu para o indicador 117 , com $15,8 \%$ de sinalizações para muito elevado e 18,4\% para baixo. O valor de 18,4\% foi utilizado como referência, concluindo-se que existia necessidade de formação em relação ao indicador que apresentasse sinalizações superiores ao valor de 18,4\% em nível muito baixo, baixo ou não se sentisse seguro para responder.

Revista Educação Online, Rio de Janeiro, n. 29, set-dez 2018, p.61-85 
Levando-se em conta o valor de $18,4 \%$ e o cenário apresentado na análise e discussão dos resultados, como também a literatura da especialidade, inicia-se a apresentação da necessidade de formação dos estudantes do ES.

\section{2 Pesquisa de informação científica}

A maioria dos estudantes indica ter competências em nível elevado e muito elevado. As indicações para nível muito baixo, baixo ou não se sentir seguro para responder estão abaixo de 18,4\%. Conclui-se que os estudantes se sentem confiantes nessa busca e talvez não necessitem de formação específica na dimensão pesquisa, do C1 ao C5. Essa inferência precisa ser avaliada em estudos qualitativos, que produzam dados a partir do registro de observações das estratégias adotadas e avaliar a sua qualidade e alcance.

\subsection{Seleção de informação científica}

Para C6, do 125 ao 129 , existe indicação de que não há necessidade de formação nesse campo, pois menos de 18,4\% dos estudantes percebem suas competências em nível muito baixo, baixo ou não se sentem seguros em responder. No entanto, seria também importante realizar estudos qualitativos com uma amostra de estudantes desse grupo. Há necessidade de formação para os dois indicadores I30 (26,3\%) e I31 (26,3\%) de C7 e, do mesmo modo, para I24 (23,7\%) de C6, pois mais de $18,4 \%$ dos estudantes indicam ter nível baixo de competências.

\subsection{Tratamento de informação científica}

Acerca de C8, existe necessidade de formação para 133 e 134, pois há sinalizações para nível baixo, respectivamente, de $23,7 \%$ e $28,9 \%$, ou seja, maior que $18,4 \%$. Não há necessidade de formação para os demais indicadores de C8, I32 e I35, assim como para todos de C9, C10, C11 e C12.

\subsection{Literatura consultada}

De forma resumida, dos 12 critérios e 47 indicadores, a maior parte dos estudantes consultados percebe suas competências em nível elevado e muito elevado em relação à maioria dos critérios e seus indicadores. Somente em relação 
a dois critérios e quatro indicadores, de forma próxima (I30 de C7 e I33 de C8) ou igual (I31 de C7 e I34 de C8), indicam ter nível de competências muito elevado e baixo. Apresentam necessidade de formação no que se refere a três critérios (C6, C7 e C8) e cinco indicadores (I24, I30, I31, I33 e 134). Ainda informam utilizar bem e muito bem determinadas ferramentas, como redes sociais, partilha de arquivos e partilha de vídeo ou áudio.

No prosseguimento desta análise, invoca-se a literatura consultada, em razão de os resultados obtidos apontarem que a maioria dos estudantes do ES demonstra ter competências relacionadas com a $\mathrm{LI}$, com recurso às $\mathrm{TIC}$, em nível elevado (HEAD, 2007; HEAD; MICHAEL B, 2009; LAMPERT, 2005; REMPEL; DAVIDSON, 2008; VARGHESE, 2008; WEILER, 2004). Observa-se que, ainda conforme as leituras realizadas (TIMMERS; VELDKAMP, 2011; WEILER, 2004), os estudantes tendem a sobrevalorizar suas competências, mesmo não apresentando ações eficazes que demonstrem dominar as competências que percebem ter, sendo um indicativo da necessidade de formação desses estudantes.

Eles consideram utilizar bem e muito bem determinadas ferramentas, como redes sociais, partilha de arquivos, de vídeo ou áudio. Isso vai ao encontro da literatura consultada (TABORDA, 2010), que sublinha que esses estudantes recorrem a enciclopédia on-line, como Wikipedia, aos blogues e, em menor escala, ao Twiter.

A afirmação dos estudantes sobre possuir competências de LI em nível elevado e muito elevado, e, da mesma forma, considerarem utilizar bem as ferramentas das TIC, vai ao encontro do que já foi considerado por outros autores. Entretanto, sublinha-se a necessidade de formação de estudantes nessa matéria.

Face ao exposto, observa-se que os estudantes do ES precisam de formação não somente nas competências que englobam os indicadores I24, I30, I31, I33 e I34, mas também no que diz respeito a todos os outros indicadores que compreendem as dimensões de pesquisa, seleção e tratamento de informação científica com recurso às TIC.

Revista Educação Online, Rio de Janeiro, n. 29, set-dez 2018, p.61-85 


\section{Conclusão}

Os resultados da investigação indicam que a maior parte dos estudantes consultados percebe possuir essas competências em nível elevado e muito elevado, somado que apontam saber utilizar ferramentas on-line/digitais. Entretanto, como mostrado na seção 4, esses estudantes tendem a superestimar as suas competências, no que tange ao uso da Internet para obtenção da informação científica (WEILER, 2004), nomeadamente no que diz respeito à LI (TIMMERS; VELDKAMP, 2011).

É de toda a importância que os estudantes, de todos os níveis de escolaridade, mas, principalmente, os que ingressam no ES, sejam proficientes no uso de recursos da Internet, principalmente, as ferramentas da Web 2.0 e 3.0, ao nível de pesquisa, seleção e tratamento de informação científica. A maior parte desses estudantes, ao realizar uma pesquisa, obtém sobrecarga de informação, com resultados irrelevantes ou com menor consistência téorica, o que pode gerar ansiedade e frustração (WEILER, 2004). Isso reflete a falta de conhecimento do potencial das TIC no contexto da LI (TIRADO; MUÑOZ, 2012) e a necessidade de formação nesse domínio. Embora a literatura consultada indique que os estudantes do ES sobrevalorizam as suas competências relacionadas com a LI, esses resultados não se baseiam em competências definidas por indicadores e critérios consensuais de avaliação. Será necessário, futuramente, consensuar formas de avaliar essas competências com maior precisão.

Em conformidade com os resultados obtidos a partir do questionário aplicado (seção 4), concluiu-se que os estudantes que participaram deste estudo percebem possuir competências relacionadas com a LI, com recurso às TIC, em nível elevado e muito elevado. Por outro lado, fundamentando-se na literatura consultada, pode-se questionar o alcance e a solidez dessas competências, uma vez que se pode afirmar saber realizar uma determinada tarefa, mas ter dificuldades em realizá-la na prática. Esse tema exige novas pesquisas, de natureza qualitativa, ancorada em observações.

Revista Educação Online, Rio de Janeiro, n. 29, set-dez 2018, p.61-85 


\subsection{Importância da investigação}

Bibliotecários e gestores de acervos de fontes para pesquisa das universidades deveriam considerar o uso dessas tecnologias, com a finalidade de socializar e ampliar estratégias de uso de bancos de dados de busca e tratamento de informação científica (HAZEN, 2011; MOLINA; VALDÉS, 2010; SILVA, 2013).

\subsection{Limitação}

O estudo traria subsídios mais sólidos e amplos, se tivesse sido possível aplicar o instrumento de pesquisa junto a um quantitativo maior de estudantes, incluindo outras universidades, com tarefas englobando cada indicador do referencial de avaliação. No entanto, não houve tempo útil para realizar o mencionado. Os resultados das ações dos estudantes, no desenvolvimento das tarefas, poderiam ser comparados com a percepção deles sobre as suas competências. Esse pode ser um caminho para pesquisas futuras.

É preciso levar em conta que os resultados aqui apresentados não podem ser generalizados para toda população de estudantes do ES. Entende-se que os resultados obtidos nesta investigação podem ser diferentes se aplicados a populações diferentes, no entanto, provavelmente, seriam próximos (AMADO, 2009).

\subsection{Recomendações}

É necessário repensar as ações de apoio à vida acadêmica de estudantes universitários e à formação em TIC de pós-graduandos, para atender a novas demandas provocadas pelas constantes alterações e desenvolvimento de tecnologias digitais em vários contextos, como o educacional.

Percebe-se que as discussões na academia têm sido feitas em torno de alterações de conteúdo programático, sem levar em conta um mundo infundido de tecnologia que reflete no processo de E/A. É inegável a existência do confronto diário com alterações no modo de aprender por parte dos estudantes, que recorrem ao uso de tecnologias digitais. A universidade deve estar atenta às necessidades dos estudantes, como um agente transformador e de experimentação ativa, possibilitando novas ideias a partir do desenvolvimento de pensamento crítico (SIEMENS; TITTENBERGER, 2009). O desenvolvimento da LI deve ser incluído de 
forma transversal no currículo (SCHMIDT; SANDERSON; TIN, 2016), como o evidenciado numa universidade australiana (SALISBURY; CORBIN; PESETA, 2013).

Deve-se atender às necessidades de formação de estudantes do ES, identificadas por meio de avaliações (HADJI, 1994), com base num referencial (SUNDIN, 2008). A aquisição das competências relacionadas com a LI, com recurso às TIC, deveria ser incremental, iterativa, curricular e avaliada (DEARDEN et al, 2004). Somado a isso, deve-se conscientizar os estudantes sobre os benefícios/facilidades alcançados pela utilização das TIC, no contexto da investigação científica e motivá-los para terem essas competências. Ter estas competências é um direito humano básico (DUDZIAK, 2013) para a promoção do desenvolvimento social (ALEIXO; NUNES; ISAIAS, 2012). A falta de preparo de estudantes do ES, nessa matéria, acarreta dificuldades de transferência das ações aprendidas no contexto acadêmico para o profissional (FERRAN-FERRER; MINGUILLÓN; PÉREZ-MONTORO, 2013).

Deveríamos nos preocupar também com esse contexto de desenvolvimento das TIC, usando as ferramentas da Web 2,0 (JEFFREY et al., 2011) e 3.0, por exemplo, gravando aulas em podcasts, disponibilizando conteúdos em apresentações on-line em diversos formatos, buscando alcançar um procedimento eficaz para a promoção dessas competências (HEAD; MICHAEL B, 2009; SALISBURY et al, 2012). Todavia, muitas universidades não conseguem mudar seus próprios modelos de vida, de trabalho, a fim de agregar novos valores aos contextos atuais (DUDZIAK, 2013), sem considerar que a mudança em educação é a expressão de uma vontade (PACHECO, 2011).

As competências relacionadas com a $\mathrm{LI}$, com recurso às TIC, não são aprendidas pela navegação na Internet (TIMMERS; GLAS, 2010). A diferença entre ser competente para navegar na Internet e o ser em relação à pesquisa, seleção e tratamento de informação científica é que, nessa última situação, existe um processo de transformação da informação para a criação de novos conhecimentos (CATTS; LAU, 2008).

Sublinha-se que é decorrente, para trabalhos futuros, a necessidade de analisar a confiabilidade do questionário desenvolvido/usado nesta investigação, Revista Educação Online, Rio de Janeiro, n. 29, set-dez 2018, p.61-85 
para a avaliação das competências de pesquisa, seleção e tratamento de informação científica, com recurso às TIC, de estudantes do ES, mas também os resultados da sua aplicação. Deve-se fazê-lo evoluir, atendendo ao feedback da sua utilização e à evolução das TIC (LYM et al., 2010).

\section{Referências bibliográficas}

AASL. Standards for the 21st - century learner American Association of School Librarians. Chicago: American Library Association, 2007.

ALEIXO, Carlos; NUNES, Miguel; ISAIAS, Pedro. Usability and digital inclusion: standards and guidelines. International Journal of Public Administration, v. 35, n. 3, p. 221-239, 2012.

AMADO, João Da Silva. Introdução à investigação qualitativa em educação (investigação educacional II). Coimbra: Faculdade de Psicologia e de Ciências da Educação, 2009.

BODGAN, Robert; BIKLEN, Sari. Investigação qualitativa em educação. Porto: Porto Editora S.A., 2013.

BOEKHORST, Albert K. Becoming information literate in the Netherlands. Library Review, v. 52, n. 7, p. 298-309, 2003. Disponível em:

<http://www.emeraldinsight.com/researchregister>. Acesso em: 7 jul. 2010.

CATTS, Ralph; LAU, Jesus. Towards information literacy indicators. 2008. Disponível em: <http://unesdoc.unesco.org/images/0015/001587/158723e.pdf>. Acesso em: 5 jan. 2010.

COHEN, Louis; MANION, Lawrence; MORRISON, Keith. Research methods in education. $5^{\text {th }}$ ed. Nova York: Routledge Falmer, 2005. Disponível em:

$<$ https://research-

srttu.wikispaces.com/file/view/Research+Methods+in+Education_ertu.pdf>. Acesso em: 7 jan. 2018.

CONNER, Tiffani Reneau. The relationship between self-directed learning and information literacy among adult learners in higher education. Tennessee: University of Tennessee, 2012. Disponível em: <http://trace.tennessee.edu/utk_graddiss/1516>. Acesso em: 16 fev. 2018.

DEARDEN, Richard et al. Shared vision, shared responsibility: the vertical integration of information literacy across the Zoology curriculum. 2004. Disponível em: <https://eprints.utas.edu.au/166/1/shared_vision.pdf>. Acesso em: 17 out. 2013.

DUDZIAK, Elisabeth. Competência em informação uma abordagem comunicacional constituição da área de estudos da área de estudos da competência infomidiática. São Paulo: Elizabeth Dudziac, 2013. Disponível em: <https://www.slideshare.net/elisabeth.dudziak>. Acesso em: 13 mar. 2013.

Revista Educação Online, Rio de Janeiro, n. 29, set-dez 2018, p.61-85 
ESHET-ALKALAI, Yoram. Digital literacy: a conceptual framework for survival skills in the digital era. Jl. of Educational Multimedia and Hypermedia, v. 13, n. 1, p. 93-106, 2004. Disponível em: <https://www.openu.ac.il/personal_sites/download/Digitalliteracy2004-JEMH.pdf>. Acesso em: 30 mar. 2018.

ETS. ICT literacy assessment preliminary findings. Princeton: Educational Testing Service, 2006.

FERNANDES, Domingos. Para uma teoria da avaliação no domínio das aprendizagens. Estudos em avaliação educacional, v. 19, n. 41, p. 347-372, 2008.

FERRAN-FERRER, Núria; MINGUILLÓN, Julià; PÉREZ-MONTORO, Mario. Key factors in the transfer of information-related competencies between academic, workplace, and daily life contexts. Journal of the American Society for Information Science and Technology, v. 64, n. 6, p. 1.112-1.121, 2013. Disponível em: <http://doi.wiley.com/10.1002/asi.22817>. Acesso em: 3 out. 2013.

FIGARI, Gérard. Évaluer: quel référentiel? Bruxelas: De Boeck-Wesmael s.a., 1994. HADJI, C. A avaliação, regras do jogo. Porto: Porto Editora LDA, 1994.

HAZEN, Dan. Lost in the Cloud: Research Library collections and community in the digital age. Library Resources \& Technical Services, v. 55, n. 4, p. 195-204, 2011. Disponível em:

<http://www.ala.org/ala/mgrps/divs/alcts/resources//rts/archive/recen>. Acesso em: 03 out. 2012

HEAD, Alison J. First monday. 2007. Disponível em:

<http://journals.uic.edu/ojs/index.php/fm/article/view/1998/1873>. Acesso em: 7 jul. 2010.

HEAD, Alison; MICHAEL B, Eisenberg. How college students seek information in the digital age project information literacy progress report. Washington: University of Washington, 2009. Disponível em:

<http://www.projectinfolit.org/uploads/2/7/5/4/27541717/pil_fall2009_finalv_yr1_12_2 009v2.pdf>. Acesso em: 5 jul. 2010.

HELMINEN, Päivi; KATJIHINGUA, Mbenae. Information literacy models in action mission immense. In. LIVONEN, M; HELMINEN, J; SISÄTTÖ, O (Eds.). Empowering -people: collaboration between finnish and Namibian University libraries. Tampere: Tampere University Press, 2012. p. 203-215Disponível em: <https://helda.helsinki.fi/handle/10138/40064> Acesso em: 06 jun. 2013

HILL, Manuela Magalhães; HILL, Andrew. Investigação por questionário. $2^{\mathrm{a}} \mathrm{ed}$. Lisboa: Edições Silabo, 2009.

JEFFREY, Lynn et al. Developing digital information literacy in higher education: obstacles and supports. Journal of Information Technology Education, v. 10, p. 383413, 2011.

KATZ, Irvin R. Testing information literacy in digital environments: ETS's iSkills Assessment. Information Technology and Libraries, v. 26, n. 3, p. 3-12, 2007. Disponível em: <http://ejournals.bc.edu/ojs/index.php/ital/article/view/3271>. Acesso em: 7 mar. 2010. 
LAMPERT, Lynn. Research and writing and theses - Oh My! The Journey of a collaboratively taught graduate research and writing course. Reference Librarian, v.43, n.89, p. 81-92, 2005. Disponível em:

$<$ http://search.ebscohost.com/login.aspx?direct=true\&db=ehh\&AN=17648360\&site =e host-live>. Acesso em: 3 out. 2010.

LE BOTERF, Guy. Construir as competências individuais e colectivas. Porto: ASA Editores S.A., 2005.

LOPES, Carlos; PINTO, Maria. IL-HUMASS - Instrumento de avaliação de competências em literacia da informação: um estudo de adaptação à população portuguesa (Parte I). [s. I.], 2010. Disponível em:

<http://repositorio.ispa.pt/bitstream/10400.12/200/1/ILHUMASS\%25\%0A20\%25E2\% 2580\%2593\%2520instrumento\%2520de\%2520avalia\%25C3\%25A7\%25C3\%25A3o $\% 2520 \% 252 \% 0$ A0de\%2520comptencias\%2520em\%2520literacia\%2520da\%2520inf orma\%25C3\%25A7\%25C3\%25A3o.pdf>. Acesso em: 30 nov. 2010.

LYM, Brian et al. Assessing the assessment: how institutions administered, interpreted, and used SAILS. Reference Services Review, v. 38, n. 1, p. 168-186, 2010. Disponível em:

<http://www.emeraldinsight.com/doi/10.1108/00907321011020806>. Acesso em: 9 ago. 2013.

MACKEY, Tom; JACOBSON, Trudi. Teaching Information literacy online. NealSchuman Publishers, p. 1-19, 2011. Disponível em:

<http://www.webs.uidaho.edu/info_literacy>. Acesso em: 27 set. 2013.

MESQUITA, Ana Filipa da Costa. Information literacy in higher education: the case of PhD students in the UP.Porto: Universidade do Porto, 2011. Disponível em:

$<$ http://repositorio-aberto.up.pt/bitstream/10216/61493/1/000148818.pdf >. Acesso em: 27 set. 2013.

MEULEMANS, Yvonne Nalani Meulemans. Assessment city: the past, present, and future state of information literacy assessment. College \& Undergraduate Libraries, v.9, n.2, p. 61-74, 2002. Disponível em:

<http://www.tandfonline.com/doi/abs/10.1300/J106v09n02_07>. Acesso em: 10 fev. 2010.

MOLINA, María Pinto; VALDÉS, María de las Mercedes Fernández. Alfabetización informacional, innovación y evaluación como funciones de la biblioteca universitaria del siglo XXI: visión desde un enfoque cualitativo. Ibersid: revista de sistemas de información y documentación, v. 4, p. 81-91, 2010. Disponível em:

<http://www.ibersid.eu/ojs/index.php/ibersid/article/view/3864>. Acesso em: 3 out. 2011.

PACHECO, José Augusto. Discursos e lugares das competências em contextos de educação e formação. Porto: Porto Editora LDA, 2011.

PALNI. Framework for information literacy for higher education: home. 2017.

Disponível em: <http://libguides.palni.edu/ilframework>. Acesso em: 18 fev. 2018.

PEREIRA, Fernando Antônio de Sousa. O desenvolvimento da competência informacional para o pensamento estratégico: uma experiência no ensino da

Revista Educação Online, Rio de Janeiro, n. 29, set-dez 2018, p.61-85 
administração de empresas através da simulação empresarial competitiva. Salvador: Universidade Federal da Bahia, 2011. Disponível em:

<https://repositorio.ufba.br/ri/bitstream/ri/7842/1/Dissertação Final.pdf>. Acesso em: 5 out. 2013.

PERRENOUD, Philippe. Construire des compétences, est-ce tourner le dos aux savoirs? Résonances, Mensuel de l'école valaisanne, v.3, p. 3-7, 1998. Disponível em: <http://www.unige.ch/fapse/SSE/teachers/perrenoud/>. Acesso em: 3 nov. 2010.

PINTO, Maria. An approach to the internal facet of Information Literacy using the ILHUMASS survey. Journal of Academic Librarianship, v.37, n.2, p. 145, 2011. Disponível em: <http://connection.ebscohost.com/c/articles/59830123/approachinternal-facet-informationliteracy-\%0Ausing-il-humass-survey>. Acesso em: 5 jan. 2012.

QUIVY, Raymond; CAMPENHOUDT, LUC Van. Manual de investigação em ciências sociais. $2^{a}$ ed. Lisboa: Gradiva - Publicações S. A, 2008. Disponível em:

$<$ https://madmunifacs.files.wordpress.com/2016/08/quivy-e-campenhoudt-manualde-investigac3a7c3a3o-em-cic3aancias-sociais-completo-r.pdf>. Acesso em: 7 jan. 2018.

REIS, Elizabeth. Estatística descritiva. $7^{\mathrm{a}}$ ed. Porto: Sílabo, 2008.

REMPEL, Hannah Gascho; DAVIDSON, Jeanne. Providing information literacy instruction to graduate students through literature review workshops. Issues in Science and Technology Librarianship, 2008. Disponível em: <http://www.istl.org/08winter/refereed2.html>. Acesso em: 25 fev. 2010.

SALISBURY, Fiona A. et al. Transforming information literacy conversations to enhance student learning: new curriculum dialogues. 2012. Disponível em: <http://ro.uow.edu.au/jutlp/vol9/iss3/4/>. Acesso em: 5 out. 2013.

SALISBURY, Fiona; CORBIN, Jenny; PESETA, Tai. From cornerstone to capstone: information literacy collaboration across the curriculum collaboration across the curriculum. In: TRIENNIAL IATUL CONFERENCES, 34., 2013, Cape Town, África do Sul. Anais... Cape Town: The International Association of Scientific and Technological University Libraries (IATUL), 2013. p. 15-18. Disponível em: < https://docs.lib.purdue.edu/iatul/2013/papers/20/>. Acesso em: 27 out. 2013.

SCHMIDT, Alice Hanbidge; SANDERSON, Nicole; TIN, Tony. Information literacy on the go! Adding mobile to an age old challenge. In: INTERNATIONAL CONFERENCE MOBILE LEARNING, 12., 2016, Ontario, Canadá. Anais... Ontario, Canadá: Renison University College, 2016. p.103 - 107. Disponível em:

<https://files.eric.ed.gov/fulltext/ED571443.pdf>. Acesso em: 20 fev. 2018.

SIEMENS, George; TITTENBERGER, Peter. Handbook of emerging technologies for learning. Manitoba: Universidade de Manitoba, 2009. Disponível em:

<http://umanitoba.ca/learning_technologies/cetl/HETL.pdf>. Acesso em: 6 jan. 2011.

SILVA, Diana. O acesso à informação científica e as tecnologias emergentes em meio académico: serviços e conteúdos das bibliotecas para a criação de valor. Aveiro: Universidade de Aveiro, 2013. Disponível em:

<https://www.slideshare.net/dianassilva1/ctdivilaconde?fb_action_ids=10151586878

Revista Educação Online, Rio de Janeiro, n. 29, set-dez 2018, p.61-85 
078749\&fb_action_types=slideshare\%3Afavorite\&fb_source=other_multiline\&action object_map $=\% 7 \mathrm{~B} \% 2210151586878078749 \% 22 \% 3$ A563999203639856\%7D\&action _type_map=\%7B\%2210151586878078749>. Acesso em: 1 maio. 2013.

ŠPIRANEC, Sonja; KOS, Denis. Information literacy practices and student protests: mapping community information landscapes. Information Research, v.18, n.3, p. paper C39, 2013. Disponível em: <http://www.informationr.net/ir/183/colis/paperC39.html\#.WomUPKinGUk>. Acesso em: 5 out. 2013.

SUNDIN, Olof. Negotiations on information-seeking expertise. Journal of Documentation, v.64, n.1, p. 24-44, 2008. Disponível em: <http://lup.lub.lu.se/search/ws/files/3117561/1053942.pdf>. Acesso em: 18 abr. 2011.

TABORDA, Maria João. A utilização de Internet em Portugal. Lisboa: LINI - Lisbon Internet and Networks, 2010. Disponível em:

<http://arquivo.pt/wayback/20170823011208/http://www.umic.pt/images/stories/notici as/Relatorio_LINI_UMIC_InternetPT.pdf>. Acesso em: 17 ago. 2011.

TIMMERS, Caroline F.; GLAS, Cees A. W. Developing scales for information-seeking behaviour. Journal of Documentation, v.66, n.1, p. 46-69, 2010. Disponível em: <http://www.emeraldinsight.com/doi/10.1108/00220411011016362>. Acesso em: 6 fev. 2011.

TIMMERS, Caroline; VELDKAMP, Bernard. Attention paid to feedback provided by a computer-based assessment for learning on information literacy. Computers and Education, v. 56, n. 3, p. 923-930, 2011. Disponível em:

<http://dx.doi.org/10.1016/j.compedu.2010.11.007>. Acesso em: 10 jun. 2012.

TIRADO, Alejandro Uribe; MUÑOZ, Wilson Castaño. Information literacy competency standards $|r|$ nfor higher education and their correlation with the cycle of knowledge generation. LIBER Quarterly, v.22, n.3, p. 213-239, 2012. Disponível em: <http://liber.library.uu.nl/index.php/lq/article/view/URN:NBN:NL:UI:10-1113941/8568>. Acesso em: 15 jan. 2013.

TUAMSUK, Kulthida. Information literacy instruction in thai higher education.

Procedia - Social and Behavioral Sciences, v.73, p.145-150, 2013. Disponível em: <http://linkinghub.elsevier.com/retrieve/pii/S1877042813003273>. Acesso em: 27 set. 2013.

UNESCO. Uso de TIC na educação do Brasil. Brasília: United Nations Educational, Scientific and Cultural Organization, 2017. Disponível em:

$<\mathrm{http}: / / \mathrm{www}$.unesco.org/new/pt/brasilia/communication-and-information/access-toknowledge/ict-in-education/>. Acesso em: 13 fev. 2018.

VARGHESE, Rekha Rani. User studies in the electronic environment: Review and brief analysis. The International Information \& Library Review, v. 40, n. 2, p. 83-93, 2008. Disponível em:

<http://linkinghub.elsevier.com/retrieve/pii/S105723170800012X>. Acesso em: 6 maio. 2010.

VIRKUS, Sije. About the learning object. 2009. Disponível em: <https://www.tlu.ee/ sirvir/Information and Knowledge Management/Concept of

Revista Educação Online, Rio de Janeiro, n. 29, set-dez 2018, p.61-85 
Information-related Competencies/about_the_learning_object.html>. Acesso em: 27 mar. 2010.

VIRKUS, Sirje. Page 3 / Information Literacy or Information-related Competencies. 2014. Disponível em: <http://www.tlu.ee/ sirvir/lKM/Informationrelated_competencies_or_information_literacy/page_3.html>. Acesso em: 18 fev. 2018.

WEILER, Angela. Information-seeking behavior in generation y students : motivation, critical thinking , and learning theory. 2004. Disponível em:

$<$ http://146.186.15.14/users/j/m/jmd394/saw4/infoseek/infoseekinggenx.pdf>. Acesso em: 7 jul. 2010. 